\title{
New Born Radii Deriving Method for Generalized Born Model
}

\author{
Wei Zhang, Tingjun Hou, and Xiaojie $\mathrm{Xu}^{*}$ \\ College of Chemistry and Molecule Engineering, Peking University, Beijing 100871, P. R. China
}

Received August 23, 2004

\begin{abstract}
Here we report a method to calculate Born radii, an important parameter used in a Generalized Born model. Traditional methods to derive Born radii are mostly based on a complicated formula, while our method is easier and more direct. Atoms are classified according to their atom type, and the Born radii of each type are obtained by fitting to experimental solvation free energy. The SMARTS language is used for the exact definition of atoms types, and Ullmann's subgraph isomorphism algorithm is used to deduce the environment. A generic algorithm is used for the parameter fitting because of its efficiency in searching a huge phase space, and its results are then optimized by using the conjugate gradient method. The final parameter set is fitting from a training set containing 357 molecules and is tested using a test set of 44 small organic molecules, and the average error is $0.58 \mathrm{kcal} / \mathrm{mol}$ for 36 neutral molecules and is $1.67 \mathrm{kcal} / \mathrm{mol}$ for 8 ions. The model is further tested under organic molecules, biopolymers, and a protein-inhibitor complex and yields reliable results in all these cases. This method can be used to accelerate molecular docking calculations.
\end{abstract}

\section{INTRODUCTION}

The rapid and accurate modeling of solvation is crucial to quantitatively understand the chemical and physical property of many biochemical processes. To solve this problem, both molecules ${ }^{1-3}$ and continuum models ${ }^{4-10}$ of the solvent have been developed. Explicit solvent models can reproduce many properties of the solutions, but such calculations converge very slowly because of the large number of particles involved; on the other hand, continuum medium models treat the solvent as a continuous medium surrounding the solute, thus they demand much less computational resources in principle.

Some continuum medium models treat solvation effects in two parts: electrostatic portion and vdw portion. The vdw portion is usually evaluated using surface area (SA) related methods, and in the simulation of biopolymers the electrostatic portion is usually treated using Poisson-Boltzmann (PB) equation based methods.

In the past few years the Generalized Born (GB) model has gotten more and more attention as a potienal model to predict the electrostatic portion, ${ }^{9}$ which can be thought of as an approximate solution to the PB equation. The GB model uses the following equations for the electrostatic portion of solvation free energy

$$
\begin{gathered}
\Delta G_{P}=-\frac{1}{2}\left(1-\frac{1}{\epsilon}\right) \sum_{i, j} \frac{q_{i} q_{j}}{f_{G B}} \\
f_{G B}=\left[r_{i j}^{2}=\alpha_{i} \alpha_{j} \exp \left(-r_{i j}^{2} / 4 \alpha_{i} \alpha_{j}\right)\right]^{1 / 2}
\end{gathered}
$$

where $\alpha_{i}$ and $\alpha_{j}$ are the effective Born radii of atom $i$ and atom $j$, respectively. In its original form, it is calculated by using an integral method, as the following equation:

\footnotetext{
* Corresponding author e-mail: xiaojxu@ chem.pku.edu.cn.
}

$$
\alpha_{i}^{-1}=r_{i}^{-1}-\frac{1}{4 \pi} \int_{i n} \frac{1}{r} d V
$$

In formula $3 r_{i}$ is atom's vdw radii, and $r$ is the distance. The integral method for Born radii is too time-consuming to be used in the simulation of biopolymers. Therefore, many efforts have been tried to derive Born radii analytically ${ }^{11-13}$ by simplifying formula 3 with some empirical parameters, and many of these parameters have been reported. ${ }^{14-17}$ However, we have a different idea. From formula 3, we can find out that the integral factor for $d V$ is $r^{-4}$, which means that only an atom's near neighborhood atom, usually called its atom type, can influence its Born radii. Theoretically an atom's near atom can have many combinations, but in practice the number of combinations is very limited because of a lot of chemical limitations. Since an atom's Born radii rely only on its atom type, we assume that atoms of the same type should have the same Born radii. On this assumption, we can predetermine each chemical environment a Born radii by fitting to the experimental solvation free energy, and in GB calculations we can assign this predetermined value to be atoms' Born radii according to their types.

The idea used here is not new. Most GB models employ constant terms for the pairwise descreening of close (bonded and $1-3$ ) neigbors, like the method of Hawkins et al., ${ }^{11}$ the ACE model of Schaefer and Karplus, ${ }^{12}$ or the method of Qiu et al., ${ }^{13}$ it can be thought of as a simplification of the earlier models.

Technically there are two problems that need to be taken care of to realize this method: one to find a proper mechanism for the definition of atom type, the other is to find a method to determine whether an atom is in a specified environment, which is in fact a subgraph isomorphism problem. For the description of atom type the SMARTS language is used because of its simplicity and convenience. As for the determination of atom type, we use Ullmann's algorithm, ${ }^{18}$ which is a standard solution to this problem. 
Table 1. Atom Patterns and Their Parameters

\begin{tabular}{|c|c|c|c|c|}
\hline name & SMARTS & description & occurrence & Born radii $(\AA)$ \\
\hline H.c & {$[\# 1][\# 6]$} & hydrogen connected to carbon & 2973 & 1.95 \\
\hline H.p & {$[\# 1][n, N, O, S]$} & hydrogen connected to polar atoms & 109 & 1.27 \\
\hline $\mathrm{C}$ & [\#6] & other carbon atoms & 1109 & 2.68 \\
\hline C.o & {$[\# 6]=\mathrm{O}$} & carbon double bonded to oxygen & 33 & 2.10 \\
\hline C.ar & [c] & aromatic carbon atoms & 552 & 2.13 \\
\hline C.n & {$[\mathrm{C} \wedge 3][\mathrm{n}, \mathrm{N}]$} & Sp3 carbon single bonded to nitrogen & 85 & 2.08 \\
\hline C.oo & {$[\# 6](=\mathrm{O}) \mathrm{O}$} & carbon in a carboxyl & 31 & 2.10 \\
\hline C.on & {$[\# 6](=\mathrm{O}) \mathrm{N}$} & carbon in amide & 6 & 3.07 \\
\hline N.1 & {$[\# 7 \wedge 1]$} & Sp1 nitrogen & 6 & 2.16 \\
\hline N.2 & {$[\# 7 \wedge 2]$} & $\mathrm{Sp} 2$ nitrogen & 16 & 2.03 \\
\hline N.3 & {$[\# 7 \wedge 3]$} & Sp3 nitrogen & 26 & 1.81 \\
\hline N.oo & {$[\# 7](\mathrm{O})=\mathrm{O}$} & nitrogen in nitryl & 6 & 2.66 \\
\hline N.ar & [n] & aromatic nitrogen & 33 & 1.90 \\
\hline 0.2 & {$[\# 8 \wedge 2]$} & Sp2 oxygen & 133 & 2.14 \\
\hline 0.3 & {$[\# 8 \wedge 3]$} & Sp3 oxygen & 65 & 1.81 \\
\hline $\mathrm{F}$ & [\#9] & fluorine & 85 & 1.57 \\
\hline $\mathrm{P}$ & [\#15] & phosphor & 7 & 3.00 \\
\hline S & [\#16] & sulfur & 25 & 2.23 \\
\hline $\mathrm{Cl}$ & [\#17] & chlorine & 114 & 2.42 \\
\hline $\mathrm{Br}$ & {$[\# 35]$} & bromine & 33 & 2.27 \\
\hline N.3+ & {$[\# 7 \wedge 3+]$} & Sp3 charged nitrogen & 12 & 1.51 \\
\hline N.2+ & {$[\# 7 \wedge 2+]$} & Sp2 charged nitrogen & 10 & 2.21 \\
\hline O.3- & {$[\# 8 \wedge 3-]$} & Sp3 charged oxygen & 3 & 1.60 \\
\hline O.2- & {$[\# 8 \wedge 2-]$ or $\mathrm{O}=\mathrm{C}[\mathrm{O}-]$} & oxygen in a charged carboxyl & 6 & 1.74 \\
\hline S- & [\#16-] & charged sulfur & 3 & 2.04 \\
\hline H.n3+ & {$[\# 1][\# 7 \wedge 3+]$} & hydrogen connected to $\mathrm{N} 3+$ & 28 & 1.44 \\
\hline H.n2+ & {$[\# 1][\# 7 \wedge 2+]$} & hydrogen connected to $\mathrm{N} 2+$ & 6 & 1.55 \\
\hline
\end{tabular}

The related code has been included in MHTL (Molecular Handling Template Library), ${ }^{19}$ a library developed in our lab, and can be obtained upon request.

\section{METHODOLOGY}

401 molecules whose experimental solvation energies are available were used in the fitting of parameters. The whole data set was divided into a training set of 357 molecules and a test set with 44 molecules; 30 molecules in the training set and 8 ones in the test set are ions. The energy-lowest conformer for each molecule was identified using a systematic search, which is done with the CeriusII software package distributed by Accelrys, Inc. The derived conformation was then minimized using the HF method under the basic set of 6-31G*. Atom's ESP charge was derived under the same basic set. The optimization and ESP work are done using Gaussian98. ${ }^{20}$

The GB/SA method is used to evaluate the solvation free energy of each molecule, the GB part is as described above, and the SA part for the vdw interaction is measured as the solvent accessible surface area $(\mathrm{SASA})^{21}$ with the surface tension being $0.005 \mathrm{kcal} \cdot \mathrm{mol}^{-1} \cdot \mathrm{A}^{-2}$.

The aim of the parameter fitting procedure is to find out a set of parameters that can predict solvation free energy most accurately. A two stage fitting strategy is employed here for the best results. In the first stage, the genetic algorithm (GA) has been used since its efficiency in searching a huge phase space, and then a conjugate gradient method has been used to optimize the results of a generic algorithm, since GA sometimes fails in finding a local minimum, which is the best part of a conjugate gradient method. The reason for its rare usage in parameter fitting work is because it requires the object function being a differentiable function of parameters. Fortunately, our method qualifies for this demand. This two stage fitting procedure (first GA, then $\mathrm{CG}$ ) ensures the resulting parameters to be a global minimum. The results also testify it, the fitting procedure has been repeated three times, each time GA gives different results, but after conjugate gradient optimization, the parameters turn out to be similar to each other.

Lots of atom types and their combinations were tried for the accurate evaluation of solvation free energy. The pattern set we finally use is made up of 27 patterns, and seven of them are designed for charged molecules. Patterns' definitions and their parameters are listed in Table 1. Atom type's appearance frequencies are also included for a reference.

In this work, the $\mathrm{PB} / \mathrm{SA}^{22}$ model is used to simulate solvation of some systems for a comparison with our method. In the PB/SA model, the electrostatic contribution to the solvation free energy are obtained by taking the difference between the total energy of the system obtained with $\epsilon$ int $=2$; $\epsilon \operatorname{ext}=1$ and $\epsilon \operatorname{int}=2 ; \epsilon \operatorname{ext}=78.5$. The $\mathrm{PB}$ calculation is performed using DelphiII, and van der Waals radii of atoms is taken from the PARSE parameter set. ${ }^{23}$

\section{RESULTS AND DISCUSSION}

The resulting model can predict the solvation free energy of the neutral molecules in the training set with a mean unsigned error of $0.63 \mathrm{kcal} / \mathrm{mol}$ (Table 1 in Supporting Information) and predict the charged molecules with a mean unsigned error of $1.60 \mathrm{kcal} / \mathrm{mol}$ (Table 2 in Supporting Information). The error for the 36 neutral molecules in the test set is $0.58 \mathrm{kcal} / \mathrm{mol}$ and is $1.79 \mathrm{kcal} / \mathrm{mol}$ for the left 8 ions (Table 2). We calculated the error for the neutral molecules and for the charged molecules individually since the solvation free energies of charged molecules are an average of 10 times larger than those of the neutral ones. The plots of the results are illustrated in Figure 1a,b. We have also calculated their solvation energy using the PB/SA model and Jayaram's GB parameters. The results of these two methods for small organic molecules are significantly worse than those using our method (Table 2). It is not 

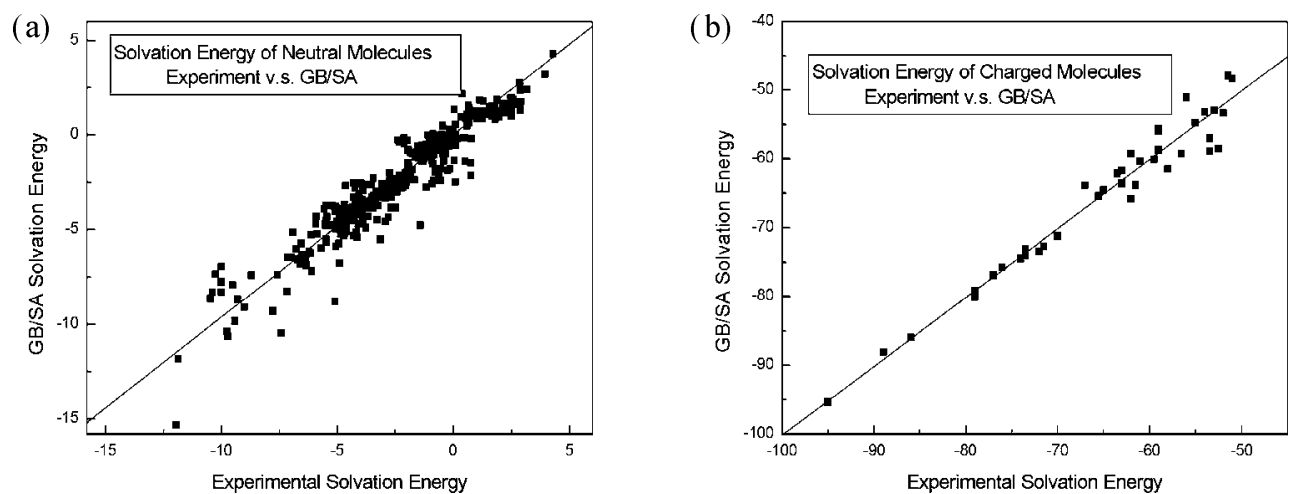

Figure 1. Experimental solvation energies of small molecules versus predicted value.

Table 2. Solvation Free Energies of Molecules in Test Set $(\mathrm{kcal} / \mathrm{mol})$

\begin{tabular}{|c|c|c|c|c|c|}
\hline no. & name & $\Delta G_{\exp }$ & $\Delta G_{\text {calc }}($ our model $)$ & $\Delta G_{\text {calc }}(\mathrm{PB} / \mathrm{SA})$ & $\Delta G_{\text {calc }}($ Jayaram' model $)$ \\
\hline A1 & $n$-pentane & 2.33 & 1.32 & 1.13 & 1.67 \\
\hline A2 & $n$-heptane & 2.62 & 1.61 & 1.29 & 1.96 \\
\hline A3 & 4-methyl-1-pentene & 1.91 & 1.85 & -1.01 & -0.19 \\
\hline A4 & 1,4-pentadiene & 0.94 & 1.24 & -2.46 & -1.29 \\
\hline A5 & butenyne & 0.04 & 0.03 & -4.01 & -3.13 \\
\hline A6 & butylbenzene & -0.40 & -1.36 & -2.16 & 0.67 \\
\hline A7 & 1,1-difluoroethane & -0.11 & -2.48 & -2.92 & -0.59 \\
\hline A8 & dichlorinedifluromethane & 1.69 & 1.01 & 1.14 & 1.68 \\
\hline A9 & 1-bromine-1,2,2,2-tetrafluoroethane & 0.52 & -1.40 & -0.86 & 2.88 \\
\hline A10 & 1,1,1,2-tetrachlorineethane & -1.15 & -0.53 & -1.53 & 1.39 \\
\hline A11 & 1,1-dichlorinebutane & -0.70 & -0.50 & -1.47 & 0.84 \\
\hline A12 & chlorinebenzene & -1.01 & -0.96 & -2.17 & 0.36 \\
\hline A13 & 1-chlorine-2-bromineethane & -1.95 & -0.80 & -2.81 & 0.28 \\
\hline A14 & 1-bromine-2-methylpropane & -0.03 & -0.01 & -1.61 & 0.17 \\
\hline A15 & o-brominecumene & -0.85 & -1.54 & -2.03 & 0.51 \\
\hline A16 & 1-butanol & -4.72 & -4.74 & -4.12 & -1.97 \\
\hline A17 & 2-methyl-1-pentanol & -3.93 & -3.81 & -3.79 & -1.51 \\
\hline A18 & 1-heptanol & -4.25 & -4.33 & -3.79 & -1.42 \\
\hline A19 & 3-cresol & -5.49 & -5.66 & -7.02 & -3.04 \\
\hline A20 & ethyl propyl ether & -1.81 & -2.15 & -0.89 & 1.25 \\
\hline A21 & 1,2-diethoxyethane & -3.53 & -3.84 & -2.16 & 1.46 \\
\hline A22 & pentanal & -3.03 & -2.84 & -4.26 & -3.16 \\
\hline A23 & m-hydroxybenzaldehyde & -9.51 & -7.91 & -10.22 & -5.51 \\
\hline A24 & cyclopentanone & -4.68 & -3.68 & -4.76 & -2.98 \\
\hline A25 & propionic acid & -6.46 & -6.56 & -8.11 & -4.80 \\
\hline A26 & isobutyl formate & -2.22 & -2.80 & -5.24 & -1.68 \\
\hline A27 & methyl propionate & -2.97 & -2.75 & -4.93 & -1.54 \\
\hline A28 & methyl hexanonate & -2.48 & -2.32 & -4.58 & -0.84 \\
\hline A29 & dimethylamine & -4.28 & -4.36 & -1.98 & -0.36 \\
\hline A30 & aniline & -5.49 & -5.53 & -7.69 & -2.31 \\
\hline A31 & pyrrolidine & -5.47 & -3.73 & -1.24 & 0.84 \\
\hline A32 & 2,4-dimethylpyridine & -4.85 & -5.23 & -4.33 & -1.44 \\
\hline A33 & 2-ethyl-3-methoypyrazine & -4.39 & -4.28 & -3.48 & -0.10 \\
\hline A34 & $\mathrm{N}^{\prime} \mathrm{N}$-dimethyl formamide & -4.90 & -4.22 & -6.81 & -4.45 \\
\hline A35 & thioanisole & -2.73 & -2.01 & -3.20 & -0.51 \\
\hline \multirow{2}{*}{ A36 } & tripropyl phosphate & -6.10 & -7.18 & -8.58 & -3.14 \\
\hline & mean unsigned error & & 0.58 & 0.73 & 1.56 \\
\hline \multicolumn{6}{|c|}{ Charged Molecules } \\
\hline A37 & $\left(\right.$ cyclo- $\left.\mathrm{C}_{6} \mathrm{H}_{11}\right) \mathrm{NH}_{3}{ }^{+}$ & -62.00 & -59.32 & -66.08 & -42.55 \\
\hline A38 & $\left(\mathrm{CH}_{3}\right)\left(\mathrm{C}_{6} \mathrm{H}_{5}\right) \mathrm{NH}_{2}^{+}$ & -56.50 & -59.25 & -62.38 & -40.48 \\
\hline A39 & $\mathrm{C}_{5} \mathrm{H}_{5} \mathrm{NH}^{+\mathrm{c}}$ & -53.50 & -57.01 & -64.08 & -46.55 \\
\hline A40 & $\left(\mathrm{CH}_{3}\right)_{2}\left(n-\mathrm{C}_{3} \mathrm{H}_{7}\right) \mathrm{NH}^{+}$ & -59.00 & -55.56 & -58.67 & -35.84 \\
\hline A41 & $\left(n-\mathrm{C}_{3} \mathrm{H}_{7}\right) \mathrm{H}_{5} \mathrm{~N}_{3} \mathrm{C}^{+\mathrm{d}}$ & -65.50 & -65.43 & -63.21 & -34.23 \\
\hline A42 & $\left(i-\mathrm{C}_{3} \mathrm{H}_{7}\right) \mathrm{H}_{5} \mathrm{~N}_{3} \mathrm{C}^{+\mathrm{d}}$ & -63.00 & -61.71 & -63.64 & -30.95 \\
\hline A43 & $\mathrm{C}_{6} \mathrm{H}_{5} \mathrm{CO}_{2}^{-}$ & -73.50 & -73.08 & -68.42 & -62.06 \\
\hline \multirow[t]{2}{*}{ A44 } & $\left(n-\mathrm{C}_{3} \mathrm{H}_{7}\right) \mathrm{S}^{-}$ & -79.00 & -79.18 & -78.81 & -71.71 \\
\hline & mean unsigned error & 0.00 & 1.79 & 3.65 & 3.63 \\
\hline
\end{tabular}

surprisingly because these methods are designed for the simulation of biopolymers and cannot ensure an accurate estimation of small organic molecules. As it can be seen, Jayaram's parameter is obtained using a relative small training set (36 molecules) obviously designed for the representation of protein. ${ }^{14}$
In Table 1, the radii for chlorine and bromine are 2.42 and 2.27 , respectively. It is abnormal because the radius for chlorine is supposed to be smaller than that of bromine. In the test set, there are 100 molecules which have chlorines and bromines in them, and most of these molecules have long hydrophobic chains. Solvation free energies of such molecules 
Table 3. Solvation Free Energies of Six Complicate Organic Molecules (kcal/mol)

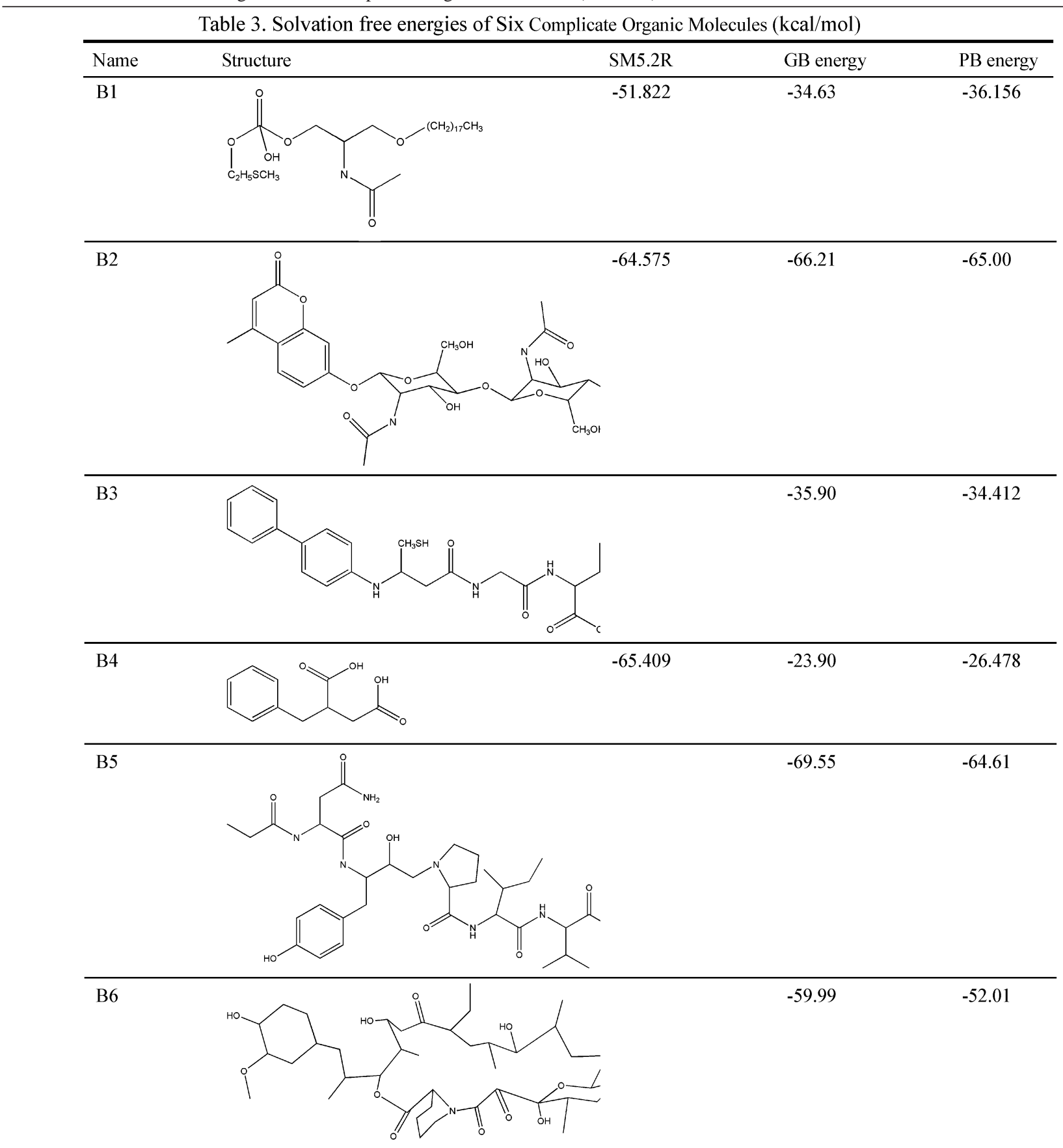

are always underestimated since they tend to aggregate together in an aqueous phase. According to eqs 1 and 2, we can imagine the radii for chlorine and bromine are both overestimated, and the radii for chlorine is overestimated more badly, because molecules with chlorine in them are much more than molecules with bromine in them, that is why the radii for chlorine is bigger than that for bromine. It can also explain the poor prediction for 1,1-difluoroethane $(-0.11 \mathrm{vs}-2.48)$ and 1-bromine-1,2,2,2-tetrafluoroethane (0.52 vs -1.40$)$ in the test set. These two molecules do not have a hydrophobic chain in them, while we still use an overestimated radii for fluorine and bromine to predict their solvation free energies. Since there are numbers of halogen atoms in the molecules, we can imagine the predicted solvation free energies for them will be greatly underestimated as well as the predicted value.
To further test our method, we have chosen six complicated molecules and calculated their GB energies using our method and their PB energies for a reference. We found the results of the two methods fit each other quite well, as shown in Table 3.

Since the GB model is mainly used in the simulations of biopolymer, we have tried our method on protein related systems. The solvation effects of these systems are hard to determine through an experimental method, so in the following cases we always use PB results as standard to test our model.

First we test our method on different proteins. Eight structures have been picked out from the Brookhaven Protein Data Bank (PDB), and their solvation energies were predicated using both the PB method and the GB method (Table 

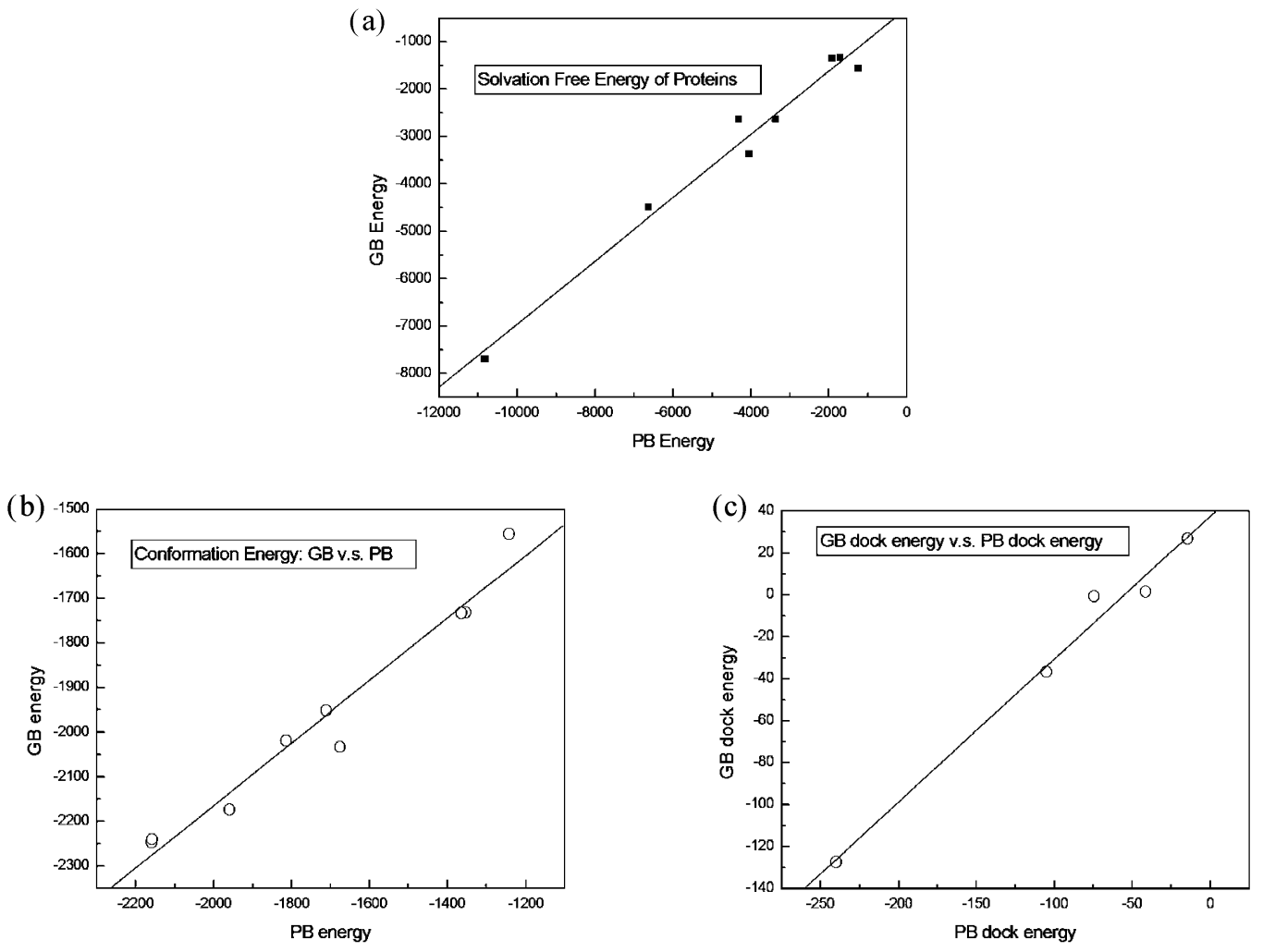

Figure 2. PB solvation free energies versus GB values: (a) different proteins, (b) different conformation of one protein, and (c) interaction energy between protein and inhibitor.

Table 4. GB Energy and PB Energy of 8 Proteins $(\mathrm{kcal} / \mathrm{mol})$

\begin{tabular}{ccr}
\hline PDB Id & PB & \multicolumn{1}{c}{ GB } \\
\hline 1ayp & -3369.0 & -4051.6 \\
1bb7 & -1349.9 & -1919.6 \\
1c3i & -7699.9 & -10825.8 \\
1cbx & -2633.3 & -4312.9 \\
1fkd & -1330.0 & -1708.1 \\
1bbs & -4488.8 & -6630.9 \\
1 gky & -2628.0 & -3372.1 \\
1ctf & -1555.6 & -1242.7 \\
\hline
\end{tabular}

Table 5. GB Energy and PB Energy of 9 Conformations of 1ctf (kcal/mol)

\begin{tabular}{ccc}
\hline Id & PB & GB \\
\hline 1 & -1242.7 & -1555.6 \\
2 & -1352.1 & -1731.5 \\
3 & -1363.5 & -1732.6 \\
4 & -1675.1 & -2033.1 \\
5 & -1711.0 & -1951.5 \\
6 & -1813.7 & -2018.6 \\
7 & -1959.1 & -2174.1 \\
8 & -2159.4 & -2247.1 \\
9 & -2158.5 & -2240.1 \\
\hline
\end{tabular}

4). It turns out that there is a good linear relation between the results of the two methods, as shown in Figure 2a.

Then we tested our method on the prediction of conformational energy of protein. We recode 9 conformations of the PDB entry 1ctf during its unfolding procedure and calculate each conformation's GB and PB energy (Table 5). There is also shown a good linear relation between our GB energy and PB energy, as illustrated in Figure $2 \mathrm{~b}$.

Last we tested this method for the prediction of the relative binding energy of protein-inhibitor. We take the lysozyme/ 4-methyl umbelliferyl chitobiose complex (PDB entry 1bb7)
Table 6. GB and PB Interaction Energy between Lysozyme and 4-Methyl Umbelliferyl Chitobiose (kcal/mol)

\begin{tabular}{rrr}
\hline Id & \multicolumn{1}{c}{ PB } & \multicolumn{1}{c}{ GB } \\
\hline 1 & -240.1 & -127.5 \\
2 & -74.3 & -0.7 \\
3 & -104.9 & -36.7 \\
4 & -41.2 & 1.4 \\
5 & -14.6 & 26.9 \\
\hline
\end{tabular}

as an example. We selected five conformations of the inhibitor in the binding pocket and predicted the GB and PB interaction energy between the inhibitor and the receptor (Table 6). The absolute value of solvation given by the two methods is different, but the two methods give the same sequence of the five conformations, and the results of the two methods show a good linear relationship with each other, as shown in Figure 2c.

According to Tables 4 and 5, the GB predicted energies are usually smaller than the PB ones. We believe it is because the Born radii of buried atoms are underestimated in our method. The fixed Born radii during the simulation may induce to the underestimation of charge-solvation forces: charged groups may shift toward the protein interior, as the self-energy of a single charge would not increase if it moved toward the interior regions, according to Onufriev et al. ${ }^{24}$ Therefore, the usage of this method in MD simulation or protein folding is rather limited, but because of its simplicity and fast we expect it can be used in molecular docking. A recent test shows that our GB method is about 1 times faster than the GB model of Hawkins et al., ${ }^{12}$ when the partial charge is given. It should be emphasized that though $6-31 \mathrm{G}^{*}$ charges are used here in this work, our method itself is charge independent; later we will derive different parameters for a 
different charge deriving method to ensure good docking results.

In this work, all the calculations were performed on a 2-CPU SGI Octane workstation. The molecule database and programs used in this work can be obtained upon request from the authors. We are now providing a patch to the AMBER source code to help AMBER users employ our method in their simulations.

\section{ACKNOWLEDGMENT}

This work is supported by Natural Science Foundation of China (No. 20375002).

Supporting Information Available: Solvation free energy of neutral molecules in training set $(\mathrm{kcal} / \mathrm{mol})$ (Table 1$)$ and solvation free energies of charged molecules in training set $(\mathrm{kcal} / \mathrm{mol})$ (Table 2). This material is available free of charge via the Internet at http://pubs.acs.org.

\section{REFERENCES AND NOTES}

(1) Rossky, P. J.; Karplus, M. Solvation. A Molecular Dynamics Study of a Dipeptide in Water. J. Am. Chem. Soc. 1979, 101, 1913-1937.

(2) Jorgensen, W. L.; Chandrasekhar, J.; Madura, J. D.; Impey, R. W.; Klein, M. L. Comparison of simple potential functions for simulating liquid water. J. Chem. Phys. 1983, 79, 926-935.

(3) Jorgensen, W. L.; Ravimohan, C. J. Monte Carlo simulation of differences in free energies of hydration. J. Chem. Phys. 1985, 83, 3050-3054.

(4) Eisenberg, D.; McLachlan, A. D. Solvation Energy in Protein Folding and Binding. Nature 1986, 319, 199-203.

(5) Ooi. T.; Oobatake, M.; Nemethy, G.; Scheraga, H. A. Accessible Surface Areas as a Measure of the Thermodynamic Parameters of Hydration of Peptides. Proc. Natl. Acad. Sci. U.S.A. 1987, 84, 30863090.

(6) Kang, Y. K.; Nemethy, G.; Scheraga, H. A. Free energy of hydration of solute molecules. 1. Improvement of the hydration shell model by exact computations of overlapping volumes. J. Phys. Chem. 1987, 91 4105-4109. 2. Application of the hydration shell model to nonionic organic molecules. 4109-4118.

(7) Kang, Y. K.; Nemethy, G.; Scheraga, H. A. Free energies of hydration of solute molecules. 3. Application of the hydration shell model to charged organic molecules J. Phys. Chem. 1987, 91, 4118-4122.

(8) Gilson, M.; Honig, B. Calculation of the total electrostatic energy of a macromolecular system: solvation energies, binding energies, and conformational analysis. Proteins 1988, 4, 7-18.

(9) Still, W. C.; Tempczyk, A.; Hawley, R. C.; Hendrickson, T. J. A General Treatment of Solvation for Molecular Mechanics. J. Am. Chem. Soc. 1990, 112, 6127-6133.

(10) Cramer, C. J.; Truhlar, D. G. General Parametrized SCF Model for Free Energies of Solvation in Aqueous Solution. J. Am. Chem. Soc.
1991, 113, 8305-8311.

(11) Qiu, D.; Shenkin, P. S.; Hollinger, F. P.; Still, W. C. The GB/SA Continuum Model for Solvation. A Fast Analytical Method for the Calculation of Approximate Born Radii. J. Phys. Chem. A 1997, 101, 3005-3014.

(12) Hawkins, G. D.; Cramer, C. J.; Truhlar, D. G. Parametrized Models of Aqueous Free Energies of Solvation Based on Pairwise Descreening of Solute Atomic Charges from a Dielectric Medium. J. Phys. Chem. 1996, 100, 19824-39.

(13) Schaefer, M.; Karplus, M. A comprehensive analytical treatment of continuum electrostatics. J. Phys. Chem. 1996, 100, 1578-1599.

(14) Jayaram, B.; Sprous, D.; Beveridge, D. L. Solvation Free Energy of Biomacromolecules: Parameters for a Modified Generalized Born Model Consistent with the AMBER Force Field. J. Phys. Chem. 1998 102, 9571-9576

(15) Zhang W.; Hou, T. J.; Qiao X. B.; Xu X. J. Parameters for the Generalized Born Model Consistent with RESP Atomic Partial Charge Assignment Protocol. J. Phys. Chem. B 2003, 107, 9071-9078.

(16) Cheng, A.; Best, S. A.; Merz, J. K. M.; Reynolds, C. H. GB/SA water model for the Merck molecular force field (MMFF). J. Mol. Graph. Mod. 2000, 18, 273-282.

(17) Zhu, J.; Shi Y. Y.; Liu, H. Y. Parametrization of a Generalized Born/ Solvent-Accessible Surface Area Model and Applications to the Simulation of Protein Dynamics. J. Phys. Chem. B 2002, 106, 48444853.

(18) Ullmann, J. R. An algorithm for subgraph isomorphism. J. As. Com Ma 1976, 23, 31-42.

(19) Lee, B.; Richards, F. M. The interpretation of protein structures: Interpretation of static accessibility. J. Mol. Biol. 1971, 55, 379-400.

(20) Zhang, W.; Hou, T.; Qiao, X.; Xu, X. Some Basic Data Structures and Algorithms for Chemical Generic Programming. J. Chem. Inf. Comput. Sci. 2004, in press.

(21) Gaussian 98 (Revision A.3), M. J. Frisch, G. W. Trucks, H. B Schlegel, G. E. Scuseria, M. A. Robb, J. R. Cheeseman, V. G. Zakrzewski, J. A. Montgomery, Jr., R. E. Stratmann, J. C. Burant, S. Dapprich, J. M. Millam, A. D. Daniels, K. N. Kudin, M. C. Strain, O. Farkas, J. Tomasi, V. Barone, M. Cossi, R. Cammi, B. Mennucci, C. Pomelli, C. Adamo, S. Clifford, J. Ochterski, G. A. Petersson, P. Y. Ayala, Q. Cui, K. Morokuma, D. K. Malick, A. D. Rabuck, K. Raghavachari, J. B. Foresman, J. Cioslowski, J. V. Ortiz, A. G. Baboul, B. B. Stefanov, G. Liu, A. Liashenko, P. Piskorz, I. Komaromi, R. Gomperts, R. L. Martin, D. J. Fox, T. Keith, M. A. Al-Laham, C. Y. Peng, A. Nanayakkara, C. Gonzalez, M. Challacombe, P. M. W. Gill, B. G. Johnson, W. Chen, M. W. Wong, J. L. Andres, M. Head-Gordon, E. S. Replogle, J. A. Pople, Gaussian, Inc., Pittsburgh, PA, 1998.

(22) Honig, B.; Sharp, K.; Yang, A.-S. Macroscopic models of aqueous solutions: Biological and chemical appications. J. Phys. Chem. 1993 , 97, 1101-1109.

(23) Sitkoff, D.; Sharp, K. A.; Honig, B. Accurate Calculation of Hydration Free Energies Using Macroscopic Solvent Models. J. Phys. Chem. 1994, 98, 1978-1988.

(24) Onufriev, A.; Case, D. A.; Bashford, D. Effective Born radii in the generalized Born approximation: The importance of being perfect. $J$. Comput. Chem. 2002, 23(14), 1297-1304.

CI0497408 